PROCEEDINGS OF THE

AMERICAN MATHEMATICAL SOCIETY

Volume 138, Number 11, November 2010, Pages 4067-4073

S 0002-9939(2010)10298-8

Article electronically published on June 18, 2010

\title{
SOME PROPERTIES OF LYAPUNOV FUNCTION SETS
}

\author{
EMMANUEL MOULAY \\ (Communicated by Peter A. Clarkson)
}

\begin{abstract}
The purpose of this paper is to study some geometrical and topological properties of Lyapunov function sets. These functions are very useful in control theory to solve stability problems. We focus our attention on the set of Lyapunov functions associated with continuous and discontinuous nonlinear systems.
\end{abstract}

\section{INTRODUCTION}

In the last few decades, there has been strong development of computational tools based on Lyapunov functions. Many such methods are based on convex optimization and solution of matrix inequalities, exploiting the fact that the set of quadratic Lyapunov functions for a given linear system is a convex cone. The structure of convex cones of matrices that are closed under matrix inversion and their connections with the algebraic Lyapunov equation is studied in [3]. In [11, the authors consider convex cones associated with quadratic Lyapunov functions for hybrid linear systems. The convex cones are also used in 8 to solve the problem of a common solution to the Lyapunov equation for $2 \times 2$ complex matrices. Nevertheless, the set of Lyapunov functions associated with nonlinear systems has not been studied in the literature. In this paper, we propose to investigate some geometrical and topological properties of the set of general smooth and nonsmooth Lyapunov functions associated with continuous and discontinuous nonlinear systems.

The paper is organized as follows. After some useful notations and definitions given in section 2, geometrical and topological properties of Lyapunov function sets associated with continuous nonlinear systems are addressed in section 3 . Section [4 is devoted to geometrical properties of Lyapunov function sets associated with discontinuous nonlinear systems. Finally, section 5 is dedicated to some possible applications.

\section{Notations AND DEFINITIONS}

A subset $C$ of a real vector space $E$ is a cone if it is closed under nonnegative scalar multiplication; i.e. for all $\alpha \in \mathbb{R}_{\geq 0}$,

$$
x \in C \quad \Rightarrow \quad \alpha x \in C .
$$

Received by the editors February 4, 2008 and, in revised form, November 23, 2009.

2010 Mathematics Subject Classification. Primary 93D20, 34D20, 37B25; Secondary 52A05.

Key words and phrases. Lyapunov functions, convex cones.

The author was supported in part by the CNRS.

(C)2010 American Mathematical Society Reverts to public domain 28 years from publication 
A cone is convex if it is closed under addition; i.e.

$$
(x, y) \in C^{2} \quad \Rightarrow \quad x+y \in C .
$$

A cone is pointed if

$$
C \cap-C=\left\{0_{E}\right\},
$$

where

$$
-C=\{x \in E:-x \in C\} .
$$

In the following, $X$ denotes an open subset of $\mathbb{R}^{n}$ containing the origin. A Fréchet space is a complete topological vector space whose topology is induced by a countable family of semi-norms. Let us recall that the set $C^{k}(X, \mathbb{R})$ is a Fréchet space for all $k \in \mathbb{N} \cup\{\infty\}$ equipped with the countable family of semi-norms

$$
\begin{array}{ll}
p_{i}(f)=\sum_{|\alpha| \leq k} \sup _{K_{i}}\left|\partial^{\alpha} f(x)\right|, \quad k \in \mathbb{N}, \\
p_{i}(f)=\sum_{|\alpha| \leq i} \sup _{K_{i}}\left|\partial^{\alpha} f(x)\right|, \quad k=\infty,
\end{array}
$$

where

$$
\begin{aligned}
\partial^{\alpha} & =\left(\frac{\partial}{\partial x_{1}}\right)^{\alpha_{1}} \ldots\left(\frac{\partial}{\partial x_{n}}\right)^{\alpha_{n}}, \quad|\alpha|=\alpha_{1}+\ldots+\alpha_{n}, \\
K_{i} & =\left\{x \in X: d\left(x, X^{c}\right) \geq \frac{1}{i}\right\} \cap\{\|x\| \leq i\} .
\end{aligned}
$$

$C^{k}(X, \mathbb{R})$ is metrizable by the distance

$$
d(f, g)=\sum_{i=1}^{+\infty} \frac{1}{2^{i}} \frac{p_{i}(f-g)}{1+p_{i}(f-g)} .
$$

Let us denote by $\mathcal{P D}(X)$ the set of all positive definite functions $V: X \rightarrow \mathbb{R}_{\geq 0}$. $\mathcal{P D}(X) \cup\{\mathbf{0}\}$ is a convex cone in the set of all functions $\mathcal{F}\left(X, \mathbb{R}^{n}\right)$, where $\mathbf{0}$ denotes the null function on $X$.

Let us recall the definition of the Dini derivative, which can be found in [4. The upper right directional Dini derivatives of a function $f: \mathbb{R}^{n} \rightarrow \mathbb{R}$ is defined by

$$
\overline{D^{+} f}(x ; v)=\limsup _{h \rightarrow 0^{+}, v^{\prime} \rightarrow v} \frac{f\left(x+h v^{\prime}\right)-f(x)}{h} .
$$

If $f$ is locally Lipschitz, we have

$$
\overline{D^{+} f}(x ; v)=\limsup _{h \rightarrow 0^{+}} \frac{f(x+h v)-f(x)}{h} ;
$$

and if $f$ is continuously differentiable, then

$$
\overline{D^{+} f}(x ; v)=\langle\nabla f(x), v\rangle .
$$




\section{Continuous systems}

Let us consider the system

$$
\dot{x}=f(x), \quad x \in X,
$$

where $f: X \rightarrow \mathbb{R}^{n}$ is a continuous function such that $f(0)=0$.

For $k \in \mathbb{N}^{*} \cup\{\infty\}$, the set of smooth Lyapunov functions for continuous systems is defined by

$$
i_{f}^{k}(X)=\left\{V \in C^{k}(X, \mathbb{R}) \cap \mathcal{P D}(X):\langle\nabla V(x), f(x)\rangle \leq 0 \quad \forall x \in X\right\},
$$

and the set of smooth strict Lyapunov functions for continuous systems is defined by

$$
\dot{L}_{f}^{k}(X)=\left\{V \in C^{k}(X, \mathbb{R}) \cap \mathcal{P D}(X):\langle\nabla V(x), f(x)\rangle<0 \quad \forall x \in X \backslash\{0\}\right\} .
$$

Moreover, we denote

$$
l_{f}^{k}(X)=i_{f}^{k}(X) \cup\{\mathbf{0}\}, \quad L_{f}^{k}(X)=\dot{L}_{f}^{k}(X) \cup\{\mathbf{0}\} .
$$

\subsection{Geometrical properties.}

Theorem 3.1. Let $k \in \mathbb{N}^{*} \cup\{\infty\}$. We have the following properties:

(1) If $V$ belongs to $i_{f}^{k}(X)$ (respectively, $\dot{L}_{f}^{k}(X)$ ), then for all $\alpha \in \mathbb{N}^{*}, V^{\alpha}$ belongs to $i_{f}^{k}(X)$ (respectively, $\dot{L}_{f}^{k}(X)$ ).

(2) $i_{f}^{k}(X)$ and $\dot{L}_{f}^{k}(X)$ are convex in $C^{k}(X, \mathbb{R})$.

(3) $l_{f}^{k}(X)$ and $L_{f}^{k}(X)$ are pointed convex cones.

Proof. Let $k \in \mathbb{N}^{*} \cup\{\infty\}$. We give the proof for $\dot{L}_{f}^{k}(X)$ and $L_{f}^{k}(X)$, but the same is true for $l_{f}^{k}(X)$ and $l_{f}^{k}(X)$. We suppose that $\dot{L}_{f}^{k}(X)$ is nonempty.

(1) If $\alpha \in \mathbb{N}^{*}, x \in X \backslash\{0\}$ and $V \in \dot{L}_{f}^{k}(X)$, we have

$$
\left\langle\nabla\left(V(x)^{\alpha}\right)(x), f(x)\right\rangle=\alpha V(x)^{\alpha-1}\langle\nabla V(x), f(x)\rangle<0 ;
$$

then $V^{\alpha} \in \dot{L}_{f}^{k}(X)$. Since $\dot{L}_{f}^{k}(X)$ is supposed to be nonempty, \# $\dot{L}_{f}^{k}(X)$ is infinite.

(2) Since $\dot{L}_{f}^{k}(X)$ is nonempty, there exists $V, W \in \dot{L}_{f}^{k}(X)$. Let $t \in[0,1]$. The function

$$
Y_{t}(x)=t V(x)+(1-t) W(x)
$$

belongs to $C^{k}(X, \mathbb{R}) \cap \mathcal{P D}$. For all $x \in X \backslash\{0\}$ and $t \in[0,1]$, we have

$$
\begin{aligned}
\left\langle\nabla Y_{t}(x), f(x)\right\rangle & =\langle\nabla[t V(x)+(1-t) W(x)](x), f(x)\rangle \\
& =t\langle\nabla V(x), f(x)\rangle+(1-t)\langle\nabla W(x), f(x)\rangle \\
& <0 .
\end{aligned}
$$

This implies that $Y_{t} \in \dot{L}_{f}^{k}(X)$ and thus that $\dot{L}_{f}^{k}(X)$ is convex.

(3) Let $V, W \in \dot{L}_{f}^{k}(X)$ and $(\alpha, \beta) \in \mathbb{R}_{\geq 0}^{2} \backslash\{(0,0)\}$. The function

$$
Y(x)=\alpha V(x)+\beta W(x)
$$

belongs to $C^{k}(X, \mathbb{R}) \cap \mathcal{P D}$. For all $x \in X \backslash\{0\}$, we have

$$
\begin{aligned}
\langle\nabla Y(x), f(x)\rangle & =\langle\nabla[\alpha V(x)+\beta W(x)](x), f(x)\rangle \\
& =\alpha\langle\nabla V(x), f(x)\rangle+\beta\langle\nabla W(x), f(x)\rangle \\
& <0 .
\end{aligned}
$$


This implies that $Y \in \dot{L}_{f}^{k}(X)$. Thus $L_{f}^{k}(X)$ is a convex cone. Since

$$
L_{f}^{k}(X) \cap-L_{f}^{k}(X)=\{\mathbf{0}\},
$$

we have that $L_{f}^{k}(X)$ is pointed.

Remark 3.2. The set $\dot{L}_{f}^{k}(X)$ is nonempty if and only if the system (3.1) is asymptotically stable (see [6, 7]). If there exists a quadratic Lyapunov function $V(x)=x^{T} P x$ for the system (3.1) where $P$ is a positive definite symmetric $n \times n$ matrix, then the sets $l_{f}^{k}(X)$ and $\dot{L}_{f}^{k}(X)$ are reduced to the finite dimensional convex cones

$$
\begin{aligned}
& i_{f}(X)=\left\{P=P^{T}: x^{T} P f(x) \leq 0 \quad \forall x \in X\right\}, \\
& \dot{L}_{f}(X)=\left\{P=P^{T}: x^{T} P f(x)<0 \quad \forall x \in X \backslash\{0\}\right\}
\end{aligned}
$$

of the set of matrices (see [3] for more details).

The set of nonsmooth Lyapunov functions for continuous systems is defined by

$$
i_{f}^{0}(X)=\left\{V \in C^{0}(X, \mathbb{R}) \cap \mathcal{P} \mathcal{D}(X): \overline{D^{+} V}(x ; f(x)) \leq 0 \quad \forall x \in X\right\},
$$

and the set of nonsmooth strict Lyapunov functions for continuous systems is defined by

$$
\dot{L}_{f}^{0}(X)=\left\{V \in C^{0}(X, \mathbb{R}) \cap \mathcal{P} \mathcal{D}(X): \overline{D^{+} V}(x ; f(x))<0 \quad \forall x \in X \backslash\{0\}\right\} .
$$

Moreover, we denote

$$
l_{f}^{0}(X)=i_{f}^{0}(X) \cup\{\mathbf{0}\}, \quad L_{f}^{0}(X)=\dot{L}_{f}^{0}(X) \cup\{\mathbf{0}\} .
$$

Theorem 3.3. We have the following properties:

(1) $i_{f}^{0}(X)$ and $\dot{L}_{f}^{0}(X)$ are convex in $C^{0}(X, \mathbb{R})$;

(2) $l_{f}^{0}(X)$ and $L_{f}^{0}(X)$ are pointed convex cones.

Proof. We give the proof for $\dot{L}_{f}^{0}(X)$ and $L_{f}^{0}(X)$, but the same is true for $i_{f}^{0}(X)$ and $l_{f}^{0}(X)$. We suppose that $\dot{L}_{f}^{0}(X)$ is nonempty.

(1) Since $\dot{L}_{f}^{0}(X)$ is nonempty, there exists $V, W \in \dot{L}_{f}^{0}(X)$. Let $t \in[0,1]$, $x \in X \backslash\{0\}$; the result follows from the inequalities

$$
\begin{aligned}
\overline{D^{+} Y_{t}}(x ; f(x)) & =\limsup _{h \rightarrow 0^{+}, v^{\prime} \rightarrow f(x)}\left[t \frac{V\left(x+h v^{\prime}\right)-V(x)}{h}+(1-t) \frac{W\left(x+h v^{\prime}\right)-W(x)}{h}\right] \\
& \leq t \overline{D^{+} V}(x ; f(x))+(1-t) \overline{D^{+} W}(x ; f(x))<0 .
\end{aligned}
$$

(2) By using the same reasoning, the result is obvious.

Property (1) of Theorem 3.1 is no more true for nonsmooth Lyapunov function sets. Indeed, in general for $\alpha \in \mathbb{N}^{*}$ we do not have

$$
\overline{D^{+} V^{\alpha}}(x ; f(x)) \leq \alpha V(x)^{\alpha-1} \overline{D^{+} V}(x ; f(x)) .
$$




\subsection{Topological properties.}

Proposition 3.4. Let $k \in \mathbb{N}^{*} \cup\{\infty\}$. The set $i_{f}^{k}(X)$ is closed in $C^{k}(X, \mathbb{R}) \cap$ $\mathcal{P D}(X)$.

Proof. Let us consider a convergent sequence $\left\{V_{n}\right\}_{n \in \mathbb{N}} \in l_{f}^{k}(X)$ such that

$$
\lim _{n \rightarrow+\infty} d\left(V_{n}, \tilde{V}\right)=0
$$

By using [13, Théorème I.2] we deduce that $\nabla V_{n}$ converges uniformly on compact subsets of $X$ to $\nabla \tilde{V}$. In particular, for all $x \in X, \nabla V_{n}(x)$ tends to $\nabla \tilde{V}(x)$. It implies that for all $x \in X$,

$$
\lim _{n \rightarrow+\infty}\left\langle\nabla V_{n}(x), f(x)\right\rangle=\langle\nabla \tilde{V}(x), f(x)\rangle \leq 0 .
$$

Thus $i_{f}^{k}(X)$ is closed in $C^{k}(X, \mathbb{R}) \cap \mathcal{P D}(X)$.

Remark 3.5. In general, we cannot prove that $\dot{L}_{f}^{k}(X)$ is open. Indeed

$$
\dot{L}_{f}^{k}(X)^{c}=\left\{V \in C^{k}(X, \mathbb{R}) \cap \mathcal{P} \mathcal{D}(X): \exists x \in X \backslash\{0\} ;\langle\nabla V(x), f(x)\rangle \geq 0\right\},
$$

and if we consider a convergent sequence $\left\{V_{n}\right\}_{n \in \mathbb{N}} \in \dot{L}_{f}^{k}(X)^{c}$, there exists a sequence $\left\{x_{n}\right\}_{n \in \mathbb{N}}$ such that $\left\langle\nabla V_{n}\left(x_{n}\right), f\left(x_{n}\right)\right\rangle \geq 0$. We cannot prove that the sequence $\nabla V_{n}\left(x_{n}\right)$ converges uniformly on compact subsets of $X$ because the sequence $\left\{x_{n}\right\}_{n \in \mathbb{N}}$ does not converge in general.

\section{Discontinuous systems}

Let $F$ be a set valued function on $X$ and consider the system

$$
\dot{x} \in F(x), \quad x \in X,
$$

where $\dot{x}$ denotes the right derivatives of $x$ and $0 \in F(0)$. We suppose that $F(x)$ is nonempty, closed, and convex for all $x \in X$ and the function $x \mapsto F(x)$ is upper semi-continuous. By virtue of the general theorem of existence for solutions of differential inclusions in [5, 1, these assumptions are sufficient for the existence for $x \in X$ of a solution $\phi^{x}(t)$ of the system (4.1) (that is an absolutely continuous function defined on an interval and satisfying the inclusion almost everywhere), defined on an interval $[0, d]$ with $d>0$, such that $\phi^{x}(0)=x$.

For $k \in \mathbb{N}^{*} \cup\{\infty\}$, the set of smooth Lyapunov functions for discontinuous systems is defined by

$$
\dot{\mathcal{L}}_{f}^{k}(X)=\left\{V \in C^{k}(X, \mathbb{R}) \cap \mathcal{P} \mathcal{D}(X): \max _{\xi \in F(x)}\langle\nabla V(x), \xi\rangle \leq 0 \quad \forall x \in X\right\},
$$

and the set of smooth strict Lyapunov functions for discontinuous systems is defined by

$$
\dot{\mathscr{L}}_{f}^{k}(X)=\left\{V \in C^{k}(X, \mathbb{R}) \cap \mathcal{P} \mathcal{D}(X): \max _{\xi \in F(x)}\langle\nabla V(x), \xi\rangle<0 \quad \forall x \in X \backslash\{0\}\right\} .
$$

The set of nonsmooth Lyapunov functions for discontinuous systems is defined by

$$
\dot{\mathcal{L}}_{f}^{0}(X)=\left\{V \in C^{0}(X, \mathbb{R}) \cap \mathcal{P} \mathcal{D}(X): \overline{D^{+} V}(x ; v) \leq 0 \quad \forall x \in X, \forall v \in F(x)\right\},
$$


and the set of nonsmooth strict Lyapunov functions for discontinuous systems is defined by

$\dot{\mathscr{L}}_{f}^{0}(X)=\left\{V \in C^{0}(X, \mathbb{R}) \cap \mathcal{P D}(X): \overline{D^{+} V}(x ; v)<0 \quad \forall x \in X \backslash\{0\}, \forall v \in F(x)\right\}$.

Moreover, we denote

$$
\begin{array}{ll}
\mathcal{L}_{f}^{k}(X)=\dot{\mathcal{L}}_{f}^{k}(X) \cup\{\mathbf{0}\}, & \mathscr{L}_{f}^{k}(X)=\dot{\mathscr{L}}_{f}^{k}(X) \cup\{\mathbf{0}\}, \\
\mathcal{L}_{f}^{0}(X)=\dot{\mathcal{L}}_{f}^{0}(X) \cup\{\mathbf{0}\}, & \mathscr{L}_{f}^{0}(X)=\dot{\mathscr{L}}_{f}^{0}(X) \cup\{\mathbf{0}\} .
\end{array}
$$

By using the same inequalities on the superior limit, we obtain the following result:

Theorem 4.1. For $k \in \mathbb{N} \cup\{\infty\}$,

(1) $\dot{\mathcal{L}}_{f}^{k}(X)$ and $\dot{\mathscr{L}}_{f}^{k}(X)$ are convex in $C^{k}(X, \mathbb{R})$;

(2) $\mathcal{L}_{f}^{k}(X)$ and $\mathscr{L}_{f}^{k}(X)$ are pointed convex cones.

Remark 4.2. The set $\dot{\mathscr{L}}_{f}^{k}(X)$ is nonempty for all $k \in \mathbb{N} \cup\{\infty\}$ if and only if the system (4.1) is strongly asymptotically stable. For a precise definition of strong stability the reader may refer to [2]. If $X=\mathbb{R}^{n}$, the Lyapunov function must also be proper; i.e. for every compact set $K \subset \mathbb{R}, V^{-1}(K)$ is compact (see [2]).

\section{Applications}

For a Lyapunov function $V: X \rightarrow \mathbb{R}_{\geq 0}$ and $\alpha \geq 0$, the level sets are given by

$$
\mathcal{V}_{\alpha}=\{x \in X: V(x) \leq \alpha\}
$$

and they define a stable region. To increase this stable region, it is not always possible to increase the $\alpha$ value due to the functioning domain of a real process. Nevertheless, it is possible to change the Lyapunov function in order to modify the geometrical form of the stable region. This can be used so as to:

- have a nonconvex region which cannot be obtained by using just a single Lyapunov function [10,

- estimate regions of asymptotic stability [12.

This is the reason why the fact that the Lyapunov function sets are convex cones is very important in order to combine several Lyapunov functions. If this result was commonly used in the literature for continuous systems with quadratic Lyapunov functions (see for instance 9] for a multi-robot system), then this paper highlights that it is also true for continuous-only Lyapunov functions and discontinuous systems.

\section{ACKNOWLEDGEMENT}

The author would like to thank Professor Samir Adly from the Department of Mathematics of the University of Limoges for many helpful discussions on convex analysis and Lyapunov functions. 


\section{REFERENCES}

1. J. P. Aubin and A. Cellina, Differential inclusions, vol. 264, Springer-Verlag, Grundlehren der Mathematischen Wissenschaften, Springer-Verlag, Berlin, 1984. MR755330 (85j:49010)

2. F. H. Clarke, Yu S. Ledyaev, and R. J. Stern, Asymptotic stability and smooth Lyapunov functions, J. Differential Equations 149 (1998), 69-114. MR,1643670 (99k:34109)

3. N. Cohen and I. Lewkowicz, Convex invertible cones and the Lyapunov equation, Linear Algebra Appl. 250 (1997), 105-131. MR 1420573 (98b:15022)

4. K. Deimling, Multivalued differential equations, De Gruyter Series in Nonlinear Analysis and Applications, 1, Walter de Gruyter, Berlin, 1992. MR1189795 (94b:34026)

5. A. F. Filippov, Differential equations with discontinuous righthand sides, Kluwer Academic Publishers, Dordrecht-Boston-London, 1988. MR.1028776 (90i:34002)

6. W. Hahn, Stability of motion, Springer-Verlag, Berlin-Heidelberg-New York, 1967. MR 0223668 (36:6716)

7. J. Kurzweil, On the inversion of Lyapunov's second theorem on stability of motion, Amer. Math. Soc. Transl. (2) 24 (1963), 19-77.

8. T. J. Laffey and H. Smigoc, Common solution to the Lyapunov equation for $2 \times 2$ complex matrices, Linear Algebra Appl. 420 (2007), no. 2-3, 609-624. MR2278236 (2007k:15020)

9. Y. Li and X. Chen, Stability on multi-robot formation with dynamic interaction topologies, IEEE International Conference on Intelligent Robots and Systems (Alberta, Canada), 2005, pp. $1325-1330$.

10. S.M. Madani-Esfahani, M. Hached, and S.H. Żak, Estimation of sliding mode domains of uncertain variable structure systems with bounded controllers, IEEE Trans. Automat. Control 35 (1990), no. 4, 446-449. MR1047998

11. O. Mason and R. Shorten, The geometry of convex cones associated with the Lyapunov inequality and the common Lyapunov function problem, Electron. J. Linear Algebra 12 (2005), 42-63. MR2139459 (2006k:37026)

12. A. N. Michel, N. R. Sarabudla, and R. K. Miller, Stability analysis of complex dynamical systems: Some computational methods, Circuits Systems Signal Process. 1 (1982), no. 2, 171-202. MR736885 (85e:34051)

13. C. Zuily and H. Queffelec, Eléments d'analyse pour l'agrégation, Masson, Paris, 1996.

Xlim (UMR-CnRS 6172), Département Signal Image Communications, Université de Poitiers, Bvd. Marie et Pierre Curie, B.P. 30179, 86962 Futuroscope Chasseneuil Cedex, FRANCE

E-mail address: emmanuel.moulay@univ-poitiers.fr 